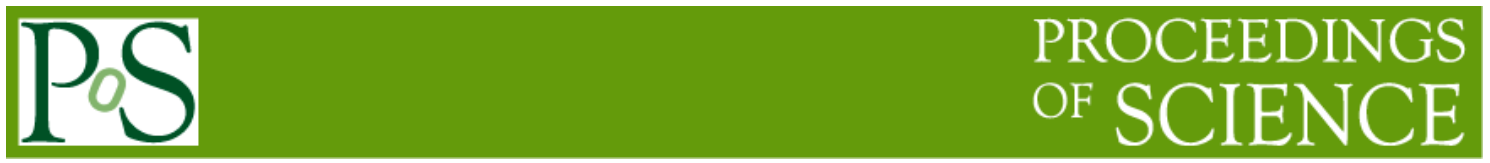

\title{
Precision tracking with small-strip Thin Gap Chamber (sTGC): from Test Beam to ATLAS NSW Upgrade
}

\author{
Yan Benhammou' \\ Tel Aviv University \\ Ramat aviv, Israel \\ E-mail: ybenhamecern.ch
}

The forthcoming luminosity upgrade of LHC to super-LHC (sLHC) will increase the expected background rate in the forward region of the ATLAS Muon Spectrometer by approximately a factor of five. Some of the present Muon Spectrometer components will fail to cope with these high rates and will have to be replaced. A development of the Thin Gap Chambers (TGC) has been tested during the last four years. The results of these tests, presented here, showed that the small strip Thin Gap Chambers (sTGC) provide a fast trigger and high precision muon tracking under sLHC conditions. In 2012, sTGC technology has been accepted as a part of the New Small Wheel (NSW).

Speaker on behalf of the ATLAS collaboration 


POS PROCEEDINGS

The European Physical Society Conference on High Energy Physics

18-24 July, 2013

Stockholm, Sweden 


\section{Introduction}

In 2018, will take place the Phase I upgrade of the LHC with a foreseen luminosity of $2.10^{34} \mathrm{~cm}^{-2} \mathrm{~s}^{-1}$; in 2022, with a new upgrade of the LHC, Phase II, the luminosity will reach $5.10^{34} \mathrm{~cm}^{-2} \mathrm{~s}^{-1}$. In order to benefit from that expected high luminosity performance, the ATLAS detector will need to be upgraded, in particular, the first station of the ATLAS muon end-cap system, the Small Wheel (SW). The New Small Wheel (NSW) will replace the SW during the long shutdown before the Phase I upgrade ${ }^{[1]}$.

On the figure 1, we can see a z-y view of one fourth of the ATLAS detector. The blue box represents the position of the actual SW. It is composed of the Cathode Strip Chambers (CSC), Thin Gap Chamber (TGC) in pink and Monitored Drift Tube (MDT) chambers in blue.

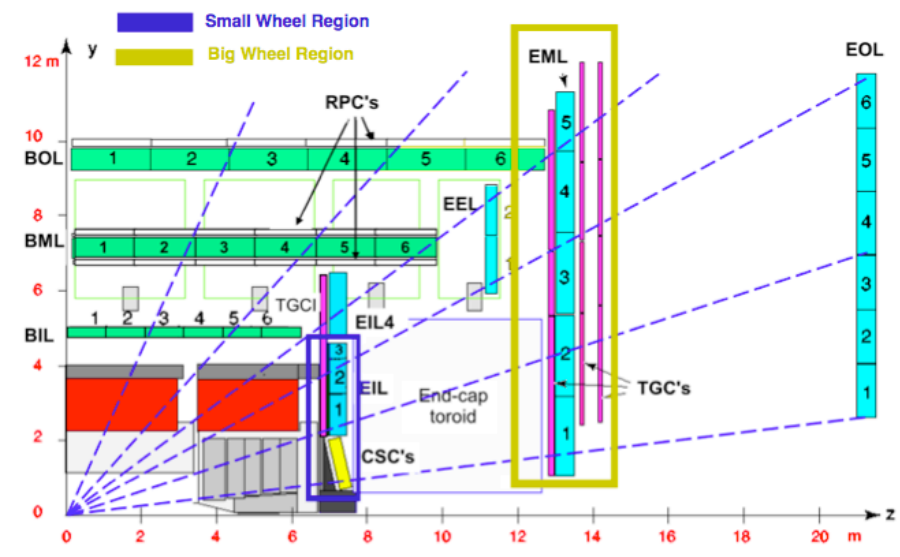

Figure 1: zy view of one fourth of the ATLAS detector

Using the simulation of the actual background cavern and extrapolating it with the data taken during the last three years, it is possible to calculate the background rate foreseen at a luminosity of $10^{34} \mathrm{~cm}^{-2} \mathrm{~s}^{-1}$ in the SW region.

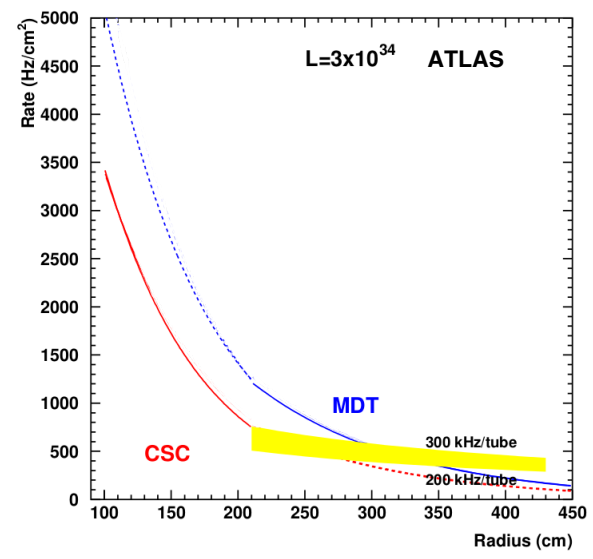

Figure 2: simulated hit rate in the $S W$ region for a luminosity of $10^{34} \mathrm{~cm}^{-2} \mathrm{~s}^{-1}$. 
The figure 2 shows the foreseen rate as a function of the radial distance of the beam. The yellow band indicates the range of tube (from MDT) rates of 200 to $300 \mathrm{kHz}$. At tube rate of $300 \mathrm{kHz}$ (the maximum rate expected at a luminosity of $10^{34} \mathrm{~cm}^{-2} \mathrm{~s}^{-1}$ ), the tube reaches hit inefficiency of about $35 \%$ and the MDT segment finding efficiency is about $90 \%$ and decreases dramatically after tube rate above $300 \mathrm{kHz}$. Hence the MDT system will be partially inefficient at the luminosities and backgrounds expected after Phase I upgrade.

It is necessary to replace the SW chambers with detectors that will allow:

- $100 \mu \mathrm{m}$ position resolution

- Online muon track reconstruction with $1 \mathrm{mrad}$ precision

To face this problem, a new type of chambers, based on the TGC technology has been developed: the small TGC (sTGC) is a multiwire chamber with $50 \mu \mathrm{m}$ diameter gold-plated tungsten wires, comprising the anode plane, located between FR4 walls coated with resistive carbon that serves as the cathode. On one side, there are strips that are perpendicular to the wires and on the opposite side, there are pads as shown on figure 3 . The spacing between the wires is $1.8 \mathrm{~mm}$ and the anode-cathode spacing is $1.4 \mathrm{~mm}$. the pitch strip is $3.2 \mathrm{~mm}$ (much smaller than the pitch of the ATLAS TGC). The operational gas is a mixture of $55 \% \mathrm{CO} 2$ and $45 \%$ n-pentane.

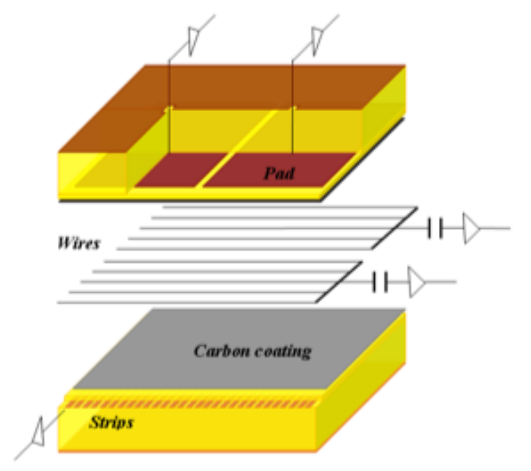

Figure 3: schematic view of the $s T G C$.

A detector is composed of 4 sTGC, i.e. 4 layers of pads, strips and wires. The pads are used to produce a 3 out of 4 coincidences to identify muon tracks coming from the interaction point. They are also used to determine which strips are to be readout to obtain a precise measurement of the position of the muon.

\section{Muon test beam results}

2.1 Test beam setup

A Typical test beam setup is described on the figure 4. It is composed of two overlapping scintillators read by photomultipliers to trigger the signal. Two monitor chambers (which are two small sTGC) are used to track the muons and, in between, the sTGC quadruplets to test. 


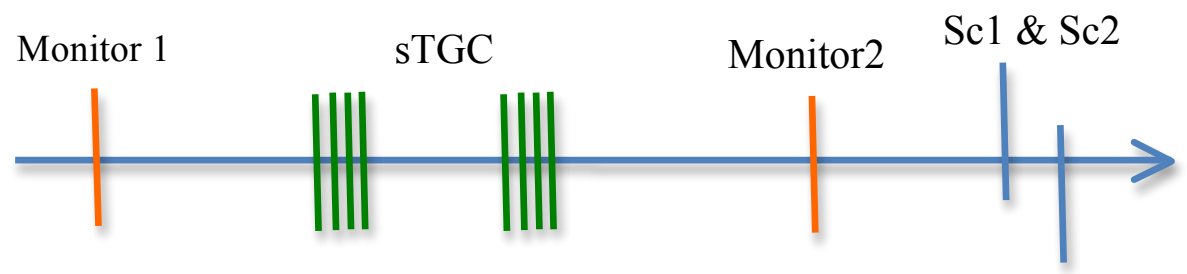

Figure 4: schematic view of the test beam line.

The very front end chip used to read the sTGC signals was the Amplifier Shaper Discriminator ${ }^{[2]}$ (ASD) designed by KEK which has a peaking time of $17 \mathrm{~ns}$ and a gain of $0.8 \mathrm{~V} / \mathrm{pC}$. We used a version of the ASD that allows getting both the digital and analog signals. VME modules read the signals mainly a TDC (digital signal) and a QDC (analog signal).

\subsection{Position resolution}

The hit position in each layer of the quadruplet is calculated using a Gaussian fit of the deposited charge (QDC) or arrival time (TDC). Then, using a linear fit including 3 layers, the track of the muon is fitted. In the fourth plan, the difference between the hit position and the expected muon position from the track is calculated and plotted (figure 5a). The $\sigma$ value of the Gaussian fit is defined as the position resolution. The position resolution varies between $60 \mu \mathrm{m}$ and $100 \mu \mathrm{m}^{[3]}$.
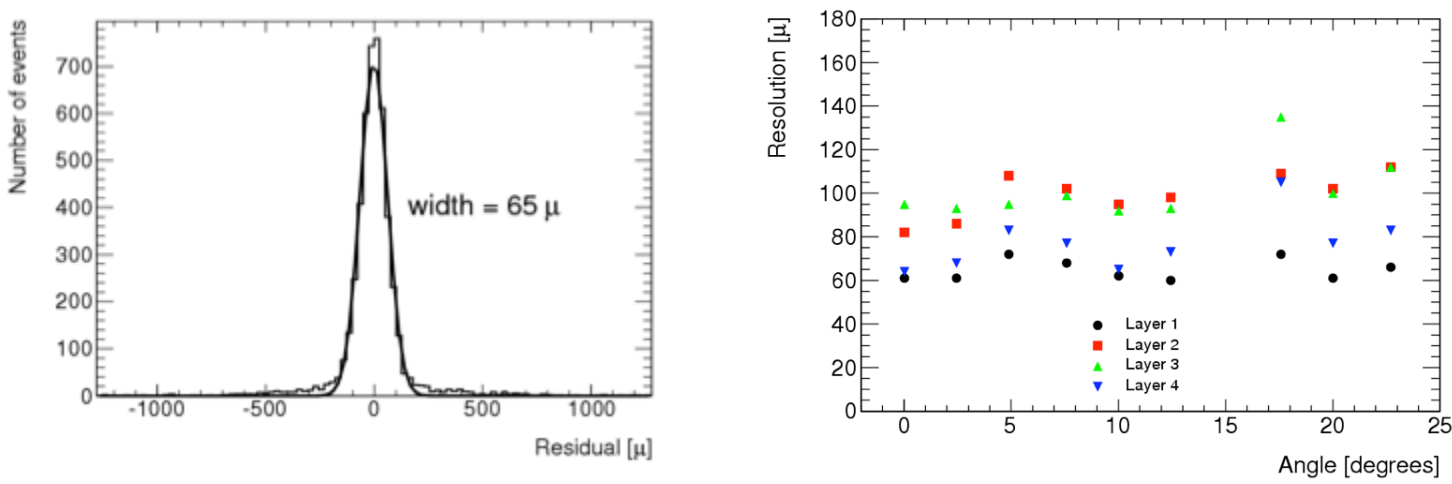

Figure 5: a) on the left, a Gaussian fit of the residual between the hit position and the expected muon track. b) on the right, the position resolution as a function of the angle for the different layers of the quadruplet.

The quadruplet has been rotated in order to measure the influence of the incident angle on the position resolution. The results (figure $5 \mathrm{~b}$ ) show that there is almost no influence of the incident angle of the muon until 20 degrees and that the quadruplet is homogenous; the position resolution between layers varies from $60 \mu \mathrm{m}$ to $105 \mu \mathrm{m}$.

\subsection{Angular resolution and pads efficiency}


A combined test beam including two quadruplets and two MDT chambers in between gave the possibility to measure the angular resolution as a function of the incident angle of the particle and the pads efficiency.
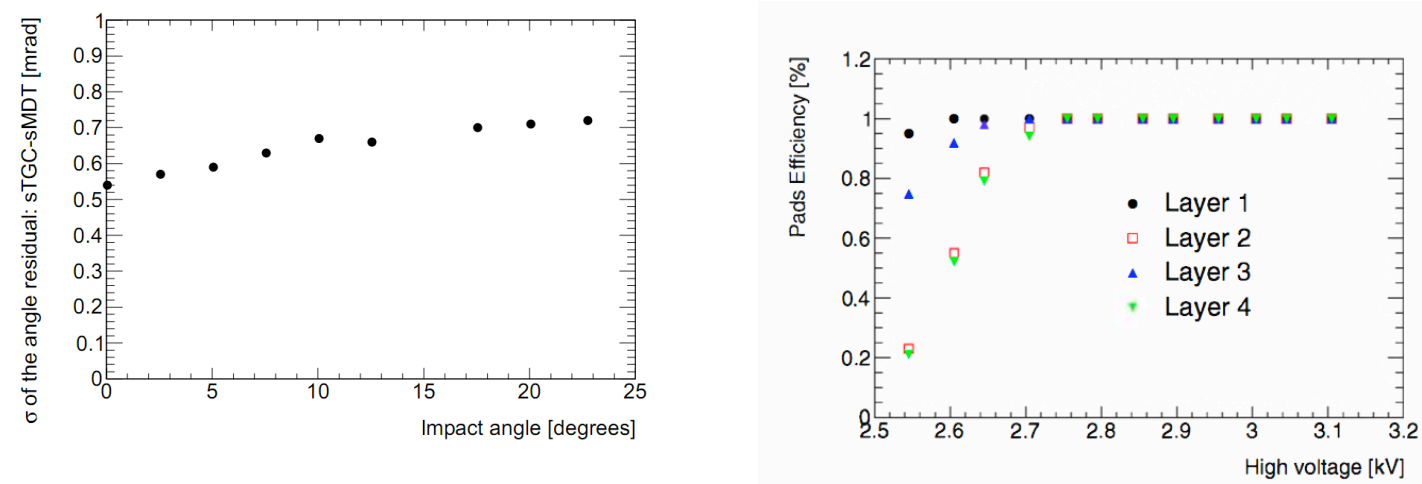

Figure 6: a) on the left, sigma of the sTGC-MDT angle residual as a function of the impact angle. b) on the right, the pads efficiency as a function of the high voltage applied on the wires

On the figure $6 \mathrm{a}$, the angular resolution has been plotted as a function of the impact angle. At angle impact around 25 degrees, the resolution is less than $1 \mathrm{mrad}$, although the beam divergence, which is around $0.5 \mathrm{mrad}$, didn't have been subtracted.

Signal coincidences from three-out-of-four sTGC layers pads were used to provide the trigger for the combined sTGC-MDT device. The efficiency of the pads as a triggering device was checked separately for each layer. The figure $6 \mathrm{~b}$ shows that the efficiency for all the layers is more than $99 \%$ above the high voltage value of $2.75 \mathrm{kV}$ (the operational high voltage for sTGC is between $2.9 \mathrm{kV}$ and $3 \mathrm{kV}$ ).

\section{Irradiation test}

\subsection{Test set-up}

For the irradiation tests, shown in figure 7, we used two pairs of TGC to trigger the cosmic muon signal and the sTGC quadruplet to be tested in between. The same front end and read out electronics as in the muons test beams was used.

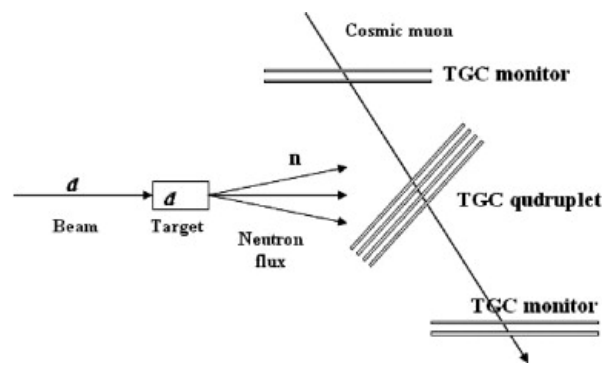

Figure 7: schematic view of the irradiation test: two pairs of TGC are used to trigger the cosmic muons and the sTGC quadruplet to be tested in between. 


\subsection{Neutron irradiation}

The sTGC have been tested at the Demokritos neutron beam in Greece. The neutrons emitted have energy between $5.5 \mathrm{MeV}$ and $6.5 \mathrm{MeV}$ and irradiated an area of about $30 \times 30 \mathrm{~cm} 2$. The procedure to determine efficiency (shown on figure 8 ) was identical as to the one described in the previous section about the position resolution i.e. fitting the cosmic muon track with the two pairs of TGC and comparing the predicted position and the measured hit in the sTGC quadruplet $^{[4]}$.

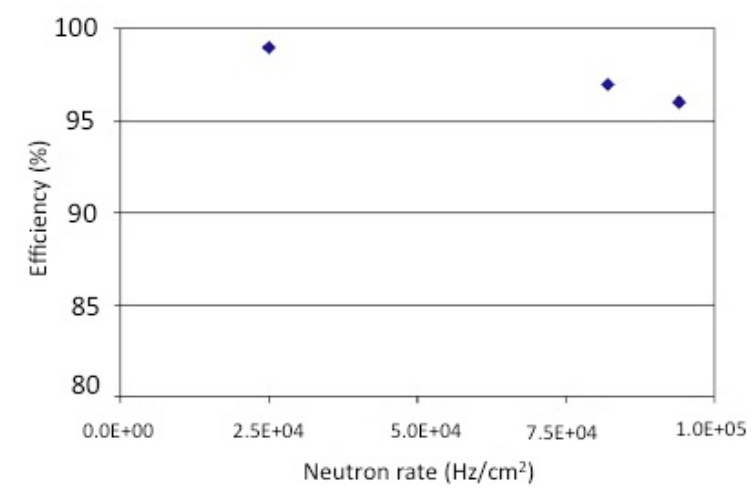

Figure 8: efficiency of the sTGC as a function of the neutron rate

The efficiency deterioration is non significant with rate below $9.10^{4} \mathrm{~Hz} / \mathrm{cm}^{2}$ (which is the neutron rate foreseen at LHC with a luminosity of $10^{35} \mathrm{~cm}^{-2} \mathrm{~s}^{-1}$ ). No sparks were observed during the 5 days period of the neutron irradiation.

\section{$3.3{ }^{60}$ Co irradiation}

The sTGC cosmic muon efficiency was also tested under a high flux of gamma photons from a $47 \mathrm{Ci}^{60} \mathrm{CO}$ source at the Soreq Nuclear Research Center, Israel. The same set up as described in figure 7 has been used and the whole sTGC quadruplet $(120 \mathrm{~cm} \times 70 \mathrm{~cm})$ was irradiated.

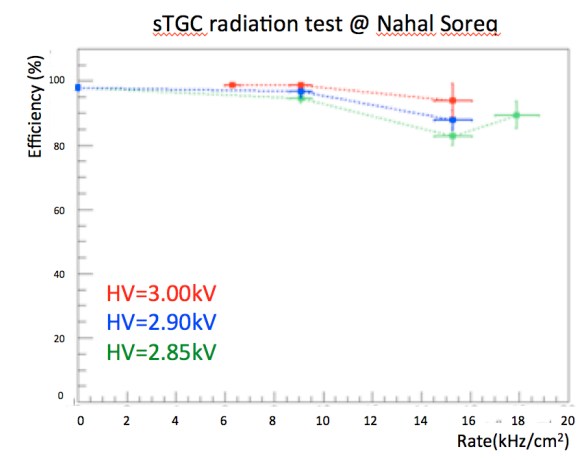

Figure 9: efficiency of the sTGC as a function of the gamma rate 
As shown in the figure 9, no efficiency deterioration was observed under a gamma flux of $2 \cdot 10^{4} \mathrm{~Hz} / \mathrm{cm} 2$. Efficiency measurements under higher fluxes up to $10^{6} \mathrm{~Hz} / \mathrm{cm} 2$ will also be performed.

\section{New front end ASIC: VMM}

In 2012, a new ASIC, the VMM ${ }^{[5]}$, has been issued, common for the sTGC and the Micromegas (the other technology that will equip the NSW with the sTGC). It provides 64 channels, an adjustable peaking time (from $25 \mathrm{~ns}$ to $200 \mathrm{~ns}$ ) and adjustable gain (from 0.5 to $9 \mathrm{~V} / \mathrm{pC}$ ), a threshold per channel and the time over threshold or time to peak value.

In the last test beam before the shut down, one layer of a sTGC quadruplet has been equipped with the VMM chip in order to perform a comparison between the ASD and VMM. On figure 10 , the results are presented.
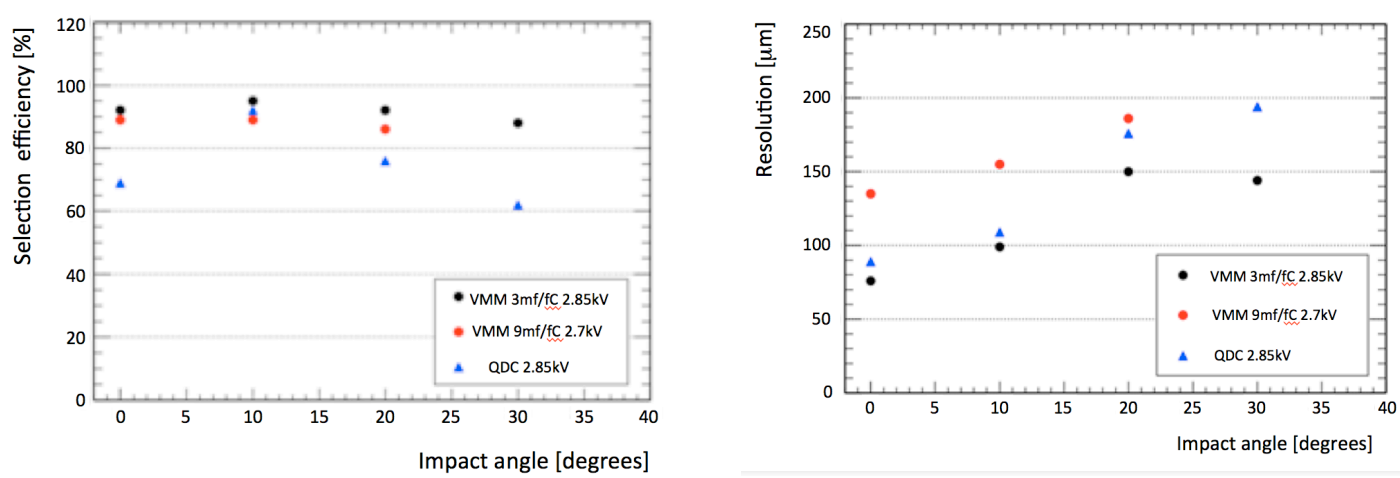

Figure 10: a) on the left, the efficiency as a function of the impact angle. b) On the right, the resolution as a function of the impact angle. Triangles are data from $A S D$, red dots from VMM $(9 \mathrm{mV} / f C)$, black dots from $V M M(3 m V / f C)$

During this test, two different configurations of the VMM chip were used: a gain of $9 \mathrm{mv} / \mathrm{fC}$ and a gain of $3 \mathrm{mv} / \mathrm{fC}$. These two configurations were compared to the ASD. On figure 10a the efficiency as a function of the impact angle has been plotted. With both gains, the efficiency with the VMM is better than the one obtained with ASD, even for angles larger than 25 degrees. The resolution as a function of the impact angle is shown on figure 10b. The VMM configuration with a larger gain gave the best results.

\section{Conclusion}

The sTGC design has been optimized during the last five years until it reaches a level which fits the LHC upgrade requirements: good position resolution and efficiency under high radiation level. With muon test beams, we reached position resolution between $60 \mu \mathrm{m}$ and $100 \mu \mathrm{m}$ depending of the impact angle and angular resolution far less than $1 \mathrm{mrad}$. The sTGC are able to support high radiation flux without loss of efficiency or position resolution. Associated with Micromegas, it will give a full redundant detector for tracking and trigger purposes. The new 
front end developed for the Micromegas and sTGC gave good results except few minor problems that will be solved in the next version of the chip. Hence the NSW will be able to provide muon trigger and position until luminosity up to $10^{35} \mathrm{~cm}^{-2} \mathrm{~s}^{-1}$.

\section{References}

[1] ATLAS collaboration, New Small Wheel Technical Design Report, CERN-LHCC-2013-006

[2] O. Sasaki and al, ASD IC for the Thin Gap Chambers in the LHC Atlas Experiment, Nuclear Science Symposium, 1998. Conference Record. 1998 IEEE

[3] Y. Benhammou and al., Test of spatial resolution and trigger efficiency of a combined Thin Gap and fast Drift Tube Chambers for high-luminosity LHC upgrades, Nuclear Science Symposium and Medical Imaging Conference (NSS/MIC), 2011 IEEE

[4] N. Amram and al., Position resolution and efficiency measurements with large scale Thin Gap Chambers for the super LHC, arXiv:1006.0135[physics.ins-det]

[5] G. de Geronimo, VMM1-An ASIC for Micropattern Detectors, IEEE Trans. Nucl. Sci. (2013) 1-

8. 